\title{
Quality of Service Management on Multimedia Data Transformation into Serial Stories Using Movement Oriented Method
}

\author{
A. Muslim \\ Etudient Doctoral Informatique \\ Le2i-UMR CNRS 5158 Université de Bourgogne \\ 9 Av. Alain Savary 21078 Dijon - France \\ Aries.muslim@etu.u-bourgogne.fr
}

\author{
A.B. Mutiara \\ Faculty Computer Science and Information Technology \\ Gunadarma University \\ Jl. Margonda Raya No. 100 Depok 16424 - Indonesia \\ amutiara@staff.gunadarma.ac.id
}

\begin{abstract}
Multimedia data transformation into serial stories or story board will help to reduce the consumption of storage media, indexing, sorting and searching system. Movement Oriented Method that is being developed changes the form of multimedia data into serial stories. Movement Oriented Method depends on the knowledge each actor who uses it. Different knowledge of each actor in the transformation process raises complex issues, such as the sequence, and the resulted story object that could become the standard. And the most fatal could be, the resulted stories does not same with the original multimedia data. To solve it, the Standard Level Knowledge (SLK) in maintaining the quality of the story could be taken. SLK is the minimum knowledge that must be owned by each actor who will perform this transformation process. Quality of Service management could be applied to assess and maintain the stability and validity of the level of the each system to SLK.
\end{abstract}

Keywords-multimedia data; serial stories; movement oriented; standard level knowledge; quality of service

\section{INTRODUCTION}

In the decades when the use of digital media is growing rapidly, both in size and type of data are not only on the text but also on the image, audio and video. Along with the increasing use of digital media, especially video, is required technical data management and retrieval of image effectively. Volume of digital video produced by the field of scientific, education, medical, industrial, and other applications available for its users increased dramatically as a result of the progress in sensor technology, the Internet and new digital video. Engineering tools annotate video with text and image search using the text-based approach. Through the description text, image can be organized by semantic hierarchy for easy navigation and search based on standard Boolean queries. Because the description text for a broad spectrum of video cannot be obtained automatically, the system mostly text-based video retrieval requires annotation manually. Indeed, manual video annotation is a difficult and expensive work for a large video database, and is often subjective, context-sensitive and not perfect [1].

There are two approaches that can be used to represent the video: (1) metadata-based and (2) content-based. Techniques required for the retrieval (query) from the two approaches which can be divided into three namely: (1) Context-based, (2) Semantic-based and (3) Content-based.

Using a content-based approach to represent a video into serial stories, the standard requires knowledge that can eliminate the error. And to keep the system knowledge, it is required of a quality level that can maintain the quality of the resulting story. The method that can keep the standard of knowledge to reaches a certain level is the quality of service management.

\section{MOVEMENT ORIENTED METHOD}

Multimedia (video) has three data elements, namely, (1) Image, (2) Vice (3) Text or character of a display to other combinations of these three elements [10]. Multimedia system design is a very complex problem, where the system complexity is growing a lot of elements on each linier changes or nonlinear of the results presentation and interaction system. Movement Oriented Design is a new paradigm that will help to manage the level of complexity in the design problem, and so that searching the multimedia data (video) is so diverse [2].

Data/video retrieval on the number of video database with a very large system using content-based experience will be a problem on identifying the time to give each frame of video files, which is a series of identifying each textual data object that will represent the video frame, where a combination of data is a metadata that will add more storage media needs that are not less [3]. Textual process of adding data to the multimedia data can be described as figure 1 .

Movement Oriented Design is a basic concept of the form of multimedia system, whether they are formal (educational) or a game (game), all of which have a certain flow of the story itself. Art establishment flow must be a story of integration of 3 scientific disciplines, namely 1) art and storytelling ability / art storytelling, 2) Cognitive psychology and 3) technology / programming [2].

At the time of this object-oriented program is a key tool in making the system of a multimedia/video that you can manipulate the object of a story, either in part or whole flow of multimedia stories. Changes can be made based on the interpretation of the respective manufacturer/user of a series of multimedia stories. These changes are very dependent on the 
level of knowledge, experience, and psychological situation of the personnel to do so. This design will produce a series of stories that come from each multimedia frame that will form the collection of data/database textual which is the identification of each frame of the overall display multimedia data. Collection of data is the alphanumeric data textual that make us easy to do with the process and indexing query during search process of data.

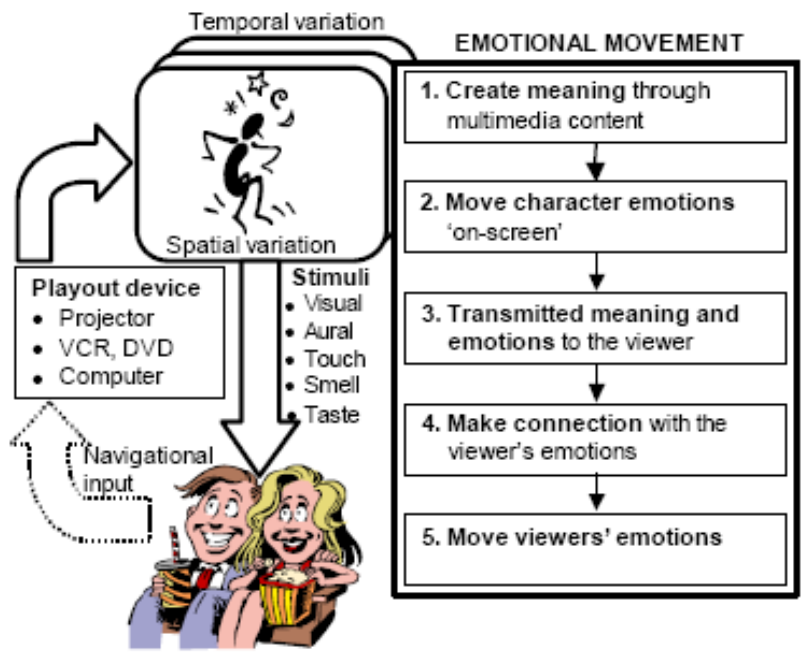

Figure 1. Multimedia Data transformation in the form of Textual Data

Quality of Service management is method to maintain the quality of service to consumers. QoS widely applied in telecommunications service and web processing. Referring to this, QoS can be used to maintain the standardization of database knowledge to the transformation of video data into serial stories, without reducing the significance with exist in the real video data. As studies on the E-Service Composer applying BPEL engine, which they express the needs of clients with their respective state standard [4].

\section{A. Problem}

- Story collection story are multimedia representation of the set of statements/character in the form of a database. It is relatively large because of the various capabilities of each of the personal view [3]. This is like the figure 2.

- $\quad$ Type of the multimedia data story of multimedia data will reflect the distinctive character, which of course will vary according to table I.

- Indexing the form of navigation and data processing work will require a relative faster speed, because each frame/stage will result in some textual statement. It requires the optimal index algorithm of data collection of stories, when it is applied on the search process or queries. An example is shown in table II. and figure 3.

- It is required the standard of knowledge base to transformation process to get story in accordance with the original video.

- It is required the management to the standardization of knowledge.

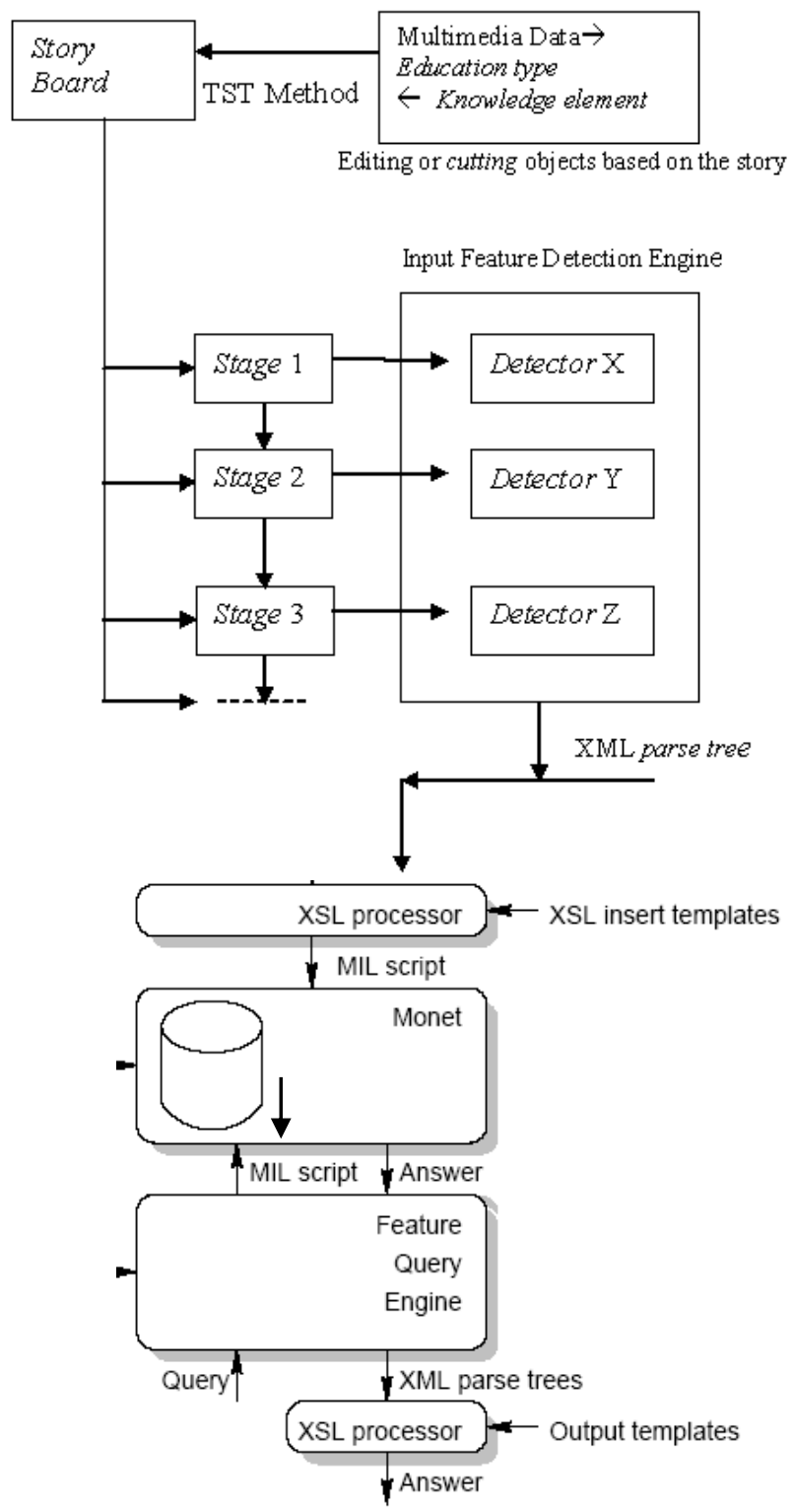

Figure 2. Diagram process data fragmentation video

TABLE I

THE CHARACTERS IN THE TABLE NEED A MULTIMEDIA DATA

\begin{tabular}{|l|l|}
\hline \multicolumn{1}{|c|}{ Story Type } & \multicolumn{1}{c|}{ Charakter Type } \\
\hline Humanistic & Human Beings \\
\hline Animated & Animation Beings \\
\hline Game & Game Beings \\
\hline Education & Knowladge elements \\
\hline Song & Word, metaphors \\
\hline Musik & Notes, Movements \\
\hline Multisensory story & " + Touch, Smell \& Taste \\
\hline Formal Story & Any of the above \\
\hline
\end{tabular}


TABLE II. MAIN PRoBlem: To EXPLAIN AND DEMONSTRATE IMPORTANT ASPECTS OF ELECTRIC CURRENT TO HIGH SCHOOL STUDENTS.

\begin{tabular}{|c|}
\hline 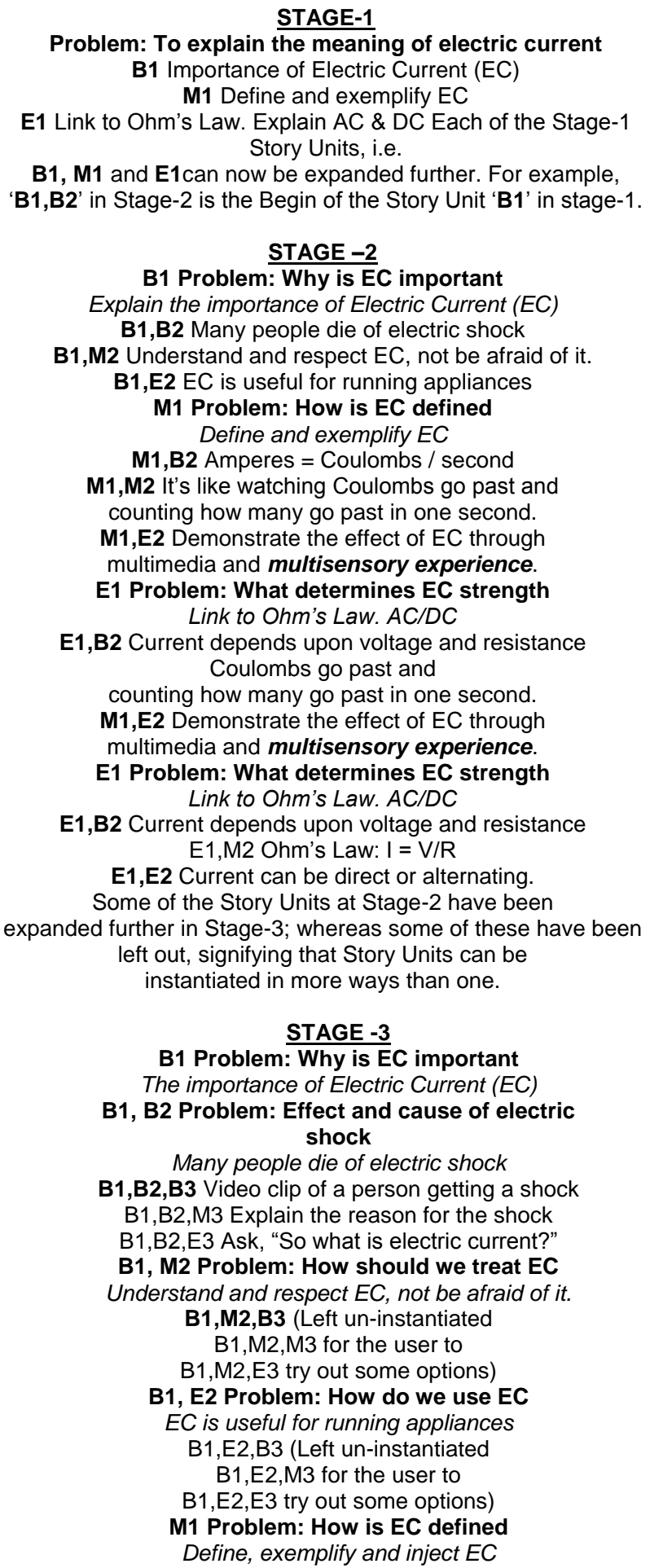 \\
\hline
\end{tabular}
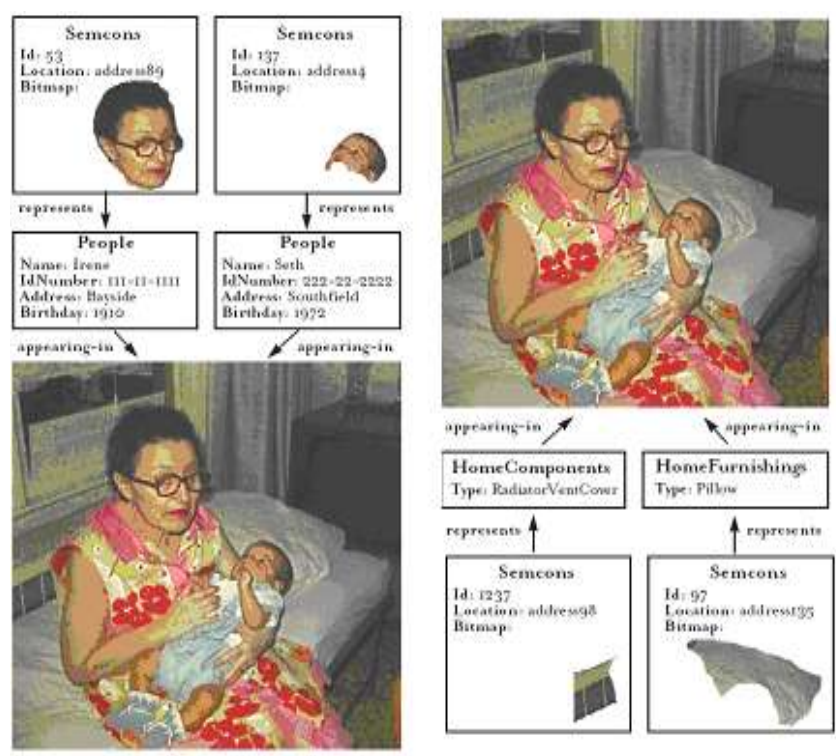

Figure 3. Content Base Method (Semcon) Identify Multimedia Data

\section{RESEARCH METHOD}

Refer to the problems in the process of change to the multimedia data in the textual data with the movement oriented method, which results in a textual database as multimedia identifying data that will be in the index, so the conducted steps in research are as following:

- We use a data-based education about robot video (MPG, AVI , MPEG3 / 4)

- Cutting the video data objects is based on the main story (Video Editing, Ulead Video Studio)

- Building a database metadata (story board, image and sound) which comes from the editing/cutting data to determine tuple video that will be the object index

- Building a video database with object oriented programming architecture based on ACOI (A System for Indexing Multimedia Object) to get tuple to process the index.

- Determining the stage for a major input in the feature detector Detection Engine.

- Making a simulation with the available databases, to design algorithms index of the overall data.

- Building a knowledge database architecture and design process of matching serial stories, image, sound and movement.

- To measure the success of the index algorithm design, we conducted the simulation / testing query data on the original database with a database that has been in the index, namely when DBA $\mathrm{T}<\mathrm{T} \mathrm{dBi}$, then the index is not successful, the algorithm index enhanced DBA $Q \gg$ $\mathrm{Q} \mathrm{dBi}$, then the index is successful. The process method with the index movement oriented to the multimedia data can be done. 
Movement Oriented as new paradigm for multimedia design, called the Movement Oriented Design, will change frame-per-frame data into a series of multimedia story (text only/stage) based on the movement, making the process identifying, indexing and searching data to be simpler. This is because the data will be textual data exploration. It is the ideal form of notice that is required by a database system, whether small or large. Multimedia data of education has a specification of a particular approach, so that changes every frame of the story plot does not become too diverse. This will facilitate the process to identify image and voice into a series of story that describes the stage of multimedia data.

Identifying a frame that has been based textual data will be easier to classify based on the limitations of the multimedia data. For example learning about the movement of robots, the classification can be drawn is that, 1) type of robot, 2) the form of a robot, 3) type of movement, 4) a lot of movement, without having to see the background, about the condition or feelings creator element. Object Oriented Programming is a technique that supports the process of classification into objects that can be used as a restriction of the index data.

Quality of Service (QOS) is the important part that must be a reference Identify when the multimedia data into textual data does not have the same level of knowledge of each personal, so that interpretation is not a frame out of the path of operation [5].

Algorithm for parallel process video/data real-time video with search range $40 \mathrm{~ms} /$ period requires a technique that is very complex, where the frame of an object is based on the main level partition needs [6]. Partition methods have been developed, namely, (1) AST - Almost Square Tiles data partitioning algorithm, (2) AST war - Almost Square Tiles data partitioning algorithm with aspect ratio, and (3) Lee-Hamdi method.

\section{Following algorithm is AST}

1) $\mathrm{k} \leftarrow$ least greater or equal square(partitions)

2) first square $\leftarrow \operatorname{squareroot}(\mathrm{k})(\mathrm{A})$

3) cols $\leftarrow$ first square

4) if (partitions is a square of an integer) rows $\leftarrow$ first square (B)

Else if ((rows-1)*cols partitions) rows $\leftarrow$ first square -1

5) $\quad$ irr col $\leftarrow$ cols $*$ rows - partitions (C)

6) $\mathrm{a} \leftarrow$ image height/rows

7) ap $\leftarrow$ image height - a * (rows -1)

8) ar $\leftarrow$ image height/num rows - 1 (D)

9) arp $\leftarrow$ image height - ar * (rows - 2)

10) $\mathrm{b} \leftarrow($ image width $/((\operatorname{ar} / \mathrm{a}) *($ cols-irr cols $)+$ irr cols $)) *$ (ar/a)

11) bp $\leftarrow$ (image width - b* (cols-irr cols) ) / irr cols (E)

12) bpp $\leftarrow$ image width - $b *$ (cols-irr cols $)$ - bp * (irr cols - 1 )

$$
\begin{aligned}
& \text { RST - regular standard ones }(\mathrm{a} * \mathrm{~b}) \text {, } \\
& \text { RET - regular excess ones (ap } * \mathrm{~b}), \\
& \text { IST - irregular standard one (bp } * \text { ar), }
\end{aligned}
$$

ICET - irregular column excess ones (bpp * ar), IRET - irregular row excess ones (bp * arp),

IRCET - irregular double excess one (bpp * arp);

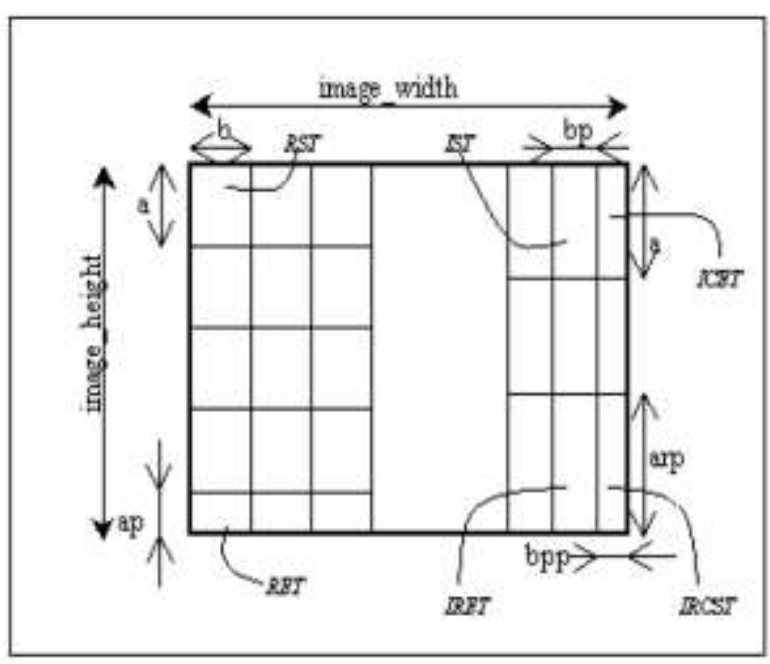

Figure 4. Picture of the position of video data on Algorithm AST

From the discussion above that the algorithm developed will be very difficult if the video database in large numbers, because will be very many objects to which the process of identification constrain index. Figure 4 shows the process identification of the image position using the AST algorithm. In the image shown in the process of collecting similar images, the regular and collected standard is called RST. For those that are not regularly, are collected into IST. So it is on both the form of row or columns.

\section{A. Indexing Multimedia Objects}

Creating Index-based multimedia objects, which provides freedom in the grammar will form a new structure on the semimain memory database system, using the derived parse tree to make the index on the source of multimedia data [7].

Detector X represents the voice data, the detector is variable among others; 1) Voters people based on gender, region of origin, age, and so forth. 2) Hearing music: music instruments, stringed instrument, musical instrument quotation, 3) natural Voters, and others.

Detector Y represents the image data, with variable detector, among others: 1) Form of the picture, 2) type of image, which can be detected with a color histogram, edge detectors and so forth. Air $\mathrm{Z}$ is the representation of text data, the variables detector more similar. 
From the above conditions it will result in the diversity of feature detection, but can be overcome by using the parse treebased object, such as the example below, figure 5 .

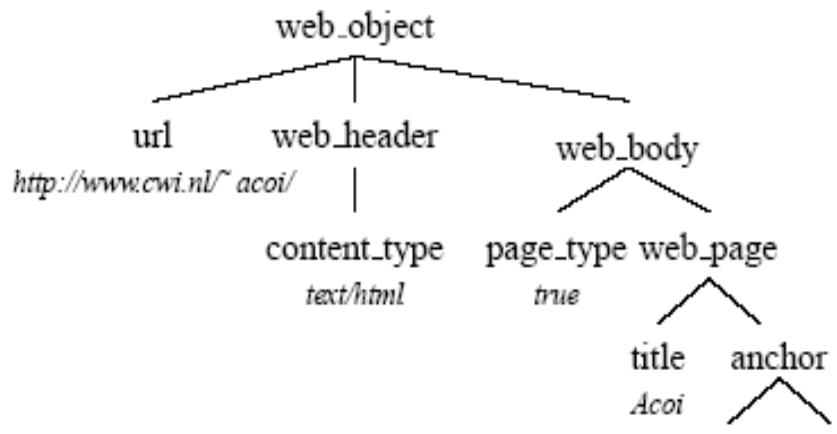

Figure 5. Parse Tree Based Object

From the parse tree approach will produce a tuple is unique, so it can be used as a variable index of the multimedia database.

\section{B. Quality of Service}

Quality of Service (QoS) is an important part that should be a reference, if the identification and transformation process multimedia data into serial stories do not have the same level of knowledge of each person. For the interpretation of a frame it is not to be of outline $[8,9]$. The transformation and identification process multimedia data into serial stories with standard level of knowledge into this process is easier and produce a collection of stories that fit with the video data. But if this done with a level of knowledge that is not standard, it can be curtained. The story is not obtained in accordance with the data video. It becomes very subjective, because each individual's knowledge becomes the size of the story.

For the process of transformation and identification of multimedia data into serial stories with a standard level of knowledge using only movement oriented method. While that does not make standard level knowledge required two additional processes, namely matching process and Quality of Service. Matching Process is to match the transformation of data with a database of knowledge and the video frame data Quality of Service is a service to provide a standard of knowledge of the data matching process before it is sent story board. With the quality of service can reduce the level subjectivities of each user.

Figure 6. shows a diagram that provides an illustration of the process of transformation and identification of multimedia data (video) into serial stories with the standard of knowledge and the non-standard of knowledge and the non-standard of knowledge.

The process that does not use a standard knowledge becomes more complex than it that uses a standard knowledge. In figure 6 it is described that there are three processes for transforming a multimedia into serial stories, 1) transformation process, 2) matching process and 3) quality of service process.

Figure 7. shows data process that is already in the matching process to carry out the quality of service. Each data derived from the matching process will be compared with a database of existing knowledge in order to become standard serial stories.

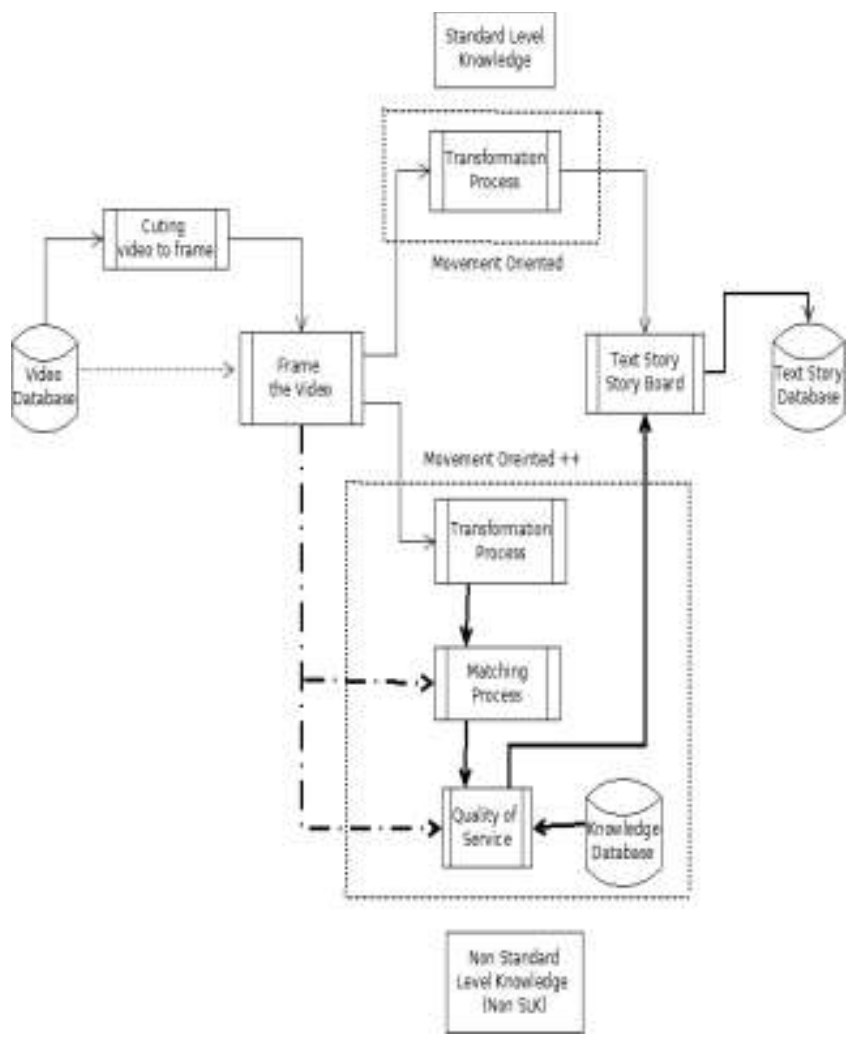

Figure 6. Movement Oriented Transformation with Quality of Service

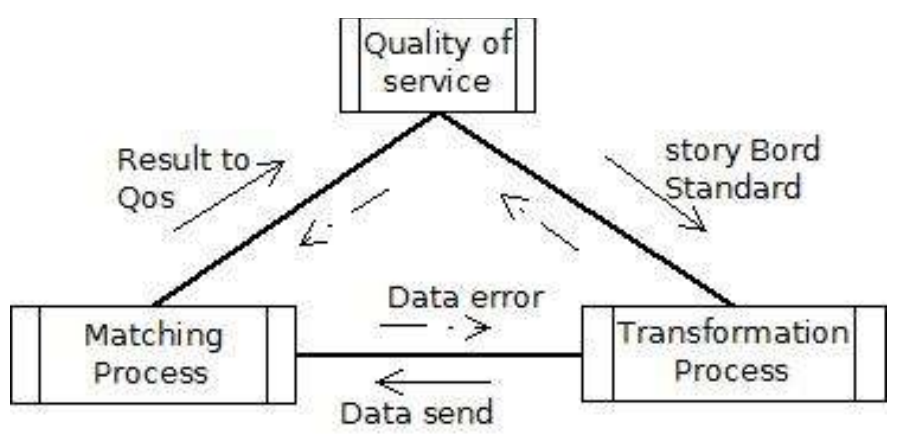

Figure 7. QoS on data Transformation and Matching Process

The result by using movement oriented with standard level knowledge is the following serial stories: robot with the background wall, swaying from side to side, motion in the legs and arms, to head downward and upward. The result of the story is very standard and does not include other element.

The result by using the movement oriented of non standard level knowledge is the following serial stories: Iron robot with eyes lit; there was a wall behind the robot, the robot moves to the left and right, rotating the robot head up and down. The transformation story approaches the desired results, but in addition there is the word "iron with his eyes lit", which is an element of user subjectivities. 
And the result by using movement oriented and non standard level knowledge and plus quality of service is the following serial stories: Robot in front of the wall, moving to the left and move to the right, on leg and arm movements, head move up and down. The transformation produces a shorter story but all of it has the standard word and knowledge.

\section{CONCLUSION AND FUTURE WORK}

The division of a series of frames from video data movement cannot be done in a partial, to avoid errors in interpretation of the relationship frame with other frames. The process of change in the video frame into a set of stories that shapes the text is a very personal knowledge each of which translated, so that it is necessary a standard knowledge on the system that was built. The process of cutting the frame is the most crucial to the next step, because the cutting process is expected to be automated (currently still rely on manual with the personal ability). Identification of sound and image are in the process of development methods, which may be able to collaborate with other research. From the results of the experiment can be conclude that the use of movement-oriented methods with a standard scenario of knowledge provides a more straightforward and assertive story. While in nonstandard knowledge and the quality of service words are more compact, dense and precise.

Development of QoS, a priority for further development, this standard provides for the cutting measurement the right. To measure the success of the design algorithm index, conducted the simulation / testing query data on the original database with a database that

\section{ACKNOWLEDGMENT}

Thanks to Prof. Kokou Yetongnon, from Le2i CNRS Université de Bourgogne, Dijon, France.

\section{REFERENCES}

[1] F. Long, Fundamentals Of Content-Based Image Retrieval, research.microsoft.com, China, 2003

[2] N. Sharda, Movement Oriented Design a New Paradigm for Multimedia Design, International Journal of Lateral Computing, Vol. 1 No. 1, May 2005

[3] W. I. Grosky, Managing Multimedia Information in Database Sistem, Communication of ACM, 1997

[4] D. Berardi, D. Calvanese, D. Giacomo, G., Lenzerini, M., Macella, M., Automatic service composition based on behavioral descriptions. International Journal of Cooperative Information systems 14 (4), 333376, 2005

[5] M.A. Phillip, C. Huntley, Dramatica: A New Theory of Story, 2001, Screenplay Systems Incorporated, California

[6] D.T. Altılar1, Y. Paker, Minimum Overhead Data Partitioning Algorithms for Parallel Video Processing, 12th International Conference on Domain Decomposition Methods, 2001

[7] 7. M. Windhouwer, A. Schmidt, M. Kersten, Acoi: A System for Indexing Multimedia Objects, CWI NL-1098 SJ Amsterdam Netherlands, 2000

[8] S. Chaudhuri, and L. Gravano, Optimizing queries over multimedia repositories. In Proceedings of SIGMOD '96 (Montreal, Canada, June 1996). ACM Press, New York, 1996.

[9] J. L. Lemke, Multiplying Meaning: Visual and Verbal Semiotics in Scientific Text, in J. R. Martin \& R. Veel (Eds.), Reading Science, London: Routledge. 1998

[10] Hanandi, M., \& Grimaldi, M. (2010). Organizational and collaborative knowledge management : a Virtual HRD model based on Web2 . 0. International Journal of Advanced Computer Science and Applications.

\section{AUTHORS PROFILE}

A Muslim, Graduate from Master Program in Information System, Gunadarma Unviversity, 1997. He is a Ph.D-Student at Groupe Database Sistem Information et Image, Le2i, UMR CNRS 5158, Faculté de Science et L'Enginer, Université de Bourgogne, Dijon, France

A.B. Mutiara is a Professor of Computer Science at Faculty of Computer Science and Information Technology, Gunadarma University 